\title{
Diagnostico nutricional del cultivo de la lima ácida Tahití [Citrus latifolia (Yu Tanaka) Tanaka] en el departamento del Tolima (Colombia)
}

\author{
Nutritional diagnosis of crops Tahiti acid lime [Citrus latifolia \\ (Yu Tanaka) Tanaka] in Tolima (Colombia)
}

\author{
Eleonora Rodríguez Polanco', Joan Sebastián Gutiérrez Díaz르, Javier Orlando Orduz Rodríguez ${ }^{3}$ \\ Recibido para publicación: Agosto 12 de 2017 - Aceptado para publicación: Mayo 24 de 2018
}

\begin{abstract}
RESUMEN
El departamento del Tolima es el principal productor de lima ácida [Citrus latifolia (Yu Tanaka) Tanaka] en Colombia. Se calcula que entre el Tolima y el Huila existen cerca de dos mil hectáreas cultivadas. El sistema productivo presenta diferencias importantes entre los productores en el manejo tecnológico con relación al riego y a la nutrición mineral, ambos relacionados significativamente con los rendimientos y calidad de la fruta. El objetivo de este estudio fue comparar la precisión, versatilidad y confiabilidad de tres métodos de interpretación de los análisis nutrimentales en el cultivo de lima Tahití. Para ello se realizaron análisis de suelo y foliares a dos plantas adultas en 20 fincas de siete municipios del departamento del Tolima cuyos resultados se interpretaron bajo el Sistema Integrado de Diagnóstico y Recomendación (DRIS), el método de Desviación del Óptimo Porcentual (DOP) y el método convencional o de Rangos de Suficiencia (RS).La información se comparó con rangos de suficiencia y se calculó la desviación del óptimo porcentual (DOP). La técnica DOP permitió diagnosticar con mayor precisión el balance nutricional en los diferentes cultivos. En los análisis de suelo, se encontró que los cultivos presentan exceso de $\mathrm{P}, \mathrm{Ca}, \mathrm{Cu}$, Fe y $\mathrm{Mn}$, mientras que tuvieron deficiencias de materia orgánica y en el $40 \%$ de las muestras de Fe y Zn. El B presentó en el $90 \%$ de las muestras un nivel óptimo. En los análisis foliares se encontró deficiencia en $\mathrm{Mg}, \mathrm{Zn}$ y $\mathrm{N}$ y excesos en $\mathrm{B}, \mathrm{Fe}, \mathrm{P}$ y $\mathrm{Cu}$. Los elementos que se encontraron en condiciones óptimas fueron $\mathrm{S}, \mathrm{Ca}$, y K. El uso conjunto de análisis de muestras foliares y de suelo permiten emitir un diagnóstico nutricional más preciso del cultivo
\end{abstract}

Palabras clave: Fincas cafeteras, agroecosistemas, cultivo, sustentabilidad y sistema agroforestal.

\begin{abstract}
In Colombia, the diversity of agroecosystems gives the coffee characteristic flavors and aromas. In the coffee crops of the Union Nariño municipality, located at $1^{\circ} 36^{\prime}$ $06^{\prime \prime}$ 'North latitude and $77^{\circ} 00^{\prime} 15^{\prime \prime}$ West longitude, the coffee activity takes place in heterogeneous farms in terms of extension, distances of sowing, applied technology, organization, family structure and fundamentally in the use and management of shade; which is why it is important to typify them by integrating the social, economic and environmental dimension and establish the differences between them, know their dynamics and determine the potential and limitations to contribute to the planning, research and organization of the coffee sector. A semistructured survey was applied to 104 coffee producers located between 1400 and 1800 meters above sea level. This information was complemented with information from institutions linked to the coffee sector. A multivariate analysis from a cluster analysis (Ward method and principal components) was applied to the variables with high correlation. Four typologies of production systems were established: coffee with shade of woody (S1), coffee semi-shade (S2), coffee-banana (S3) and the coffee without shade (S4). Within each typology, different dynamics were found in crop management practices, leading to differences in production, quality and economic yields.
\end{abstract}

Key words: Coffee farms, agroecosystems, crop sustainability, and agroforestry system.

\footnotetext{
${ }^{1}$ Ph.D. Corporación Colombiana de Investigación Agropecuaria. C.I. Nataima. El Espinal.

${ }^{2}$ I.A. Estudiante Maestría en Ciencias Agrarias con énfasis en Suelos y Aguas. Universidad Nacional de Colombia.

${ }^{3}$ Ph.D. Corporación Colombiana de Investigación Agropecuaria. C.I. La Libertad, Villavicencio.
} 


\section{INTRODUCCIÓN}

Los cítricos se cultivan en la mayor parte de las regiones tropicales y subtropicales del planeta, aunque las principales zonas productoras del mundo están ubicadas en el subtrópico entre los 15 y $35^{\circ}$ de latitud en ambos hemisferios (Jackson, 1999). Los cítricos que se comercializan en el mundo se distribuyen en seis especies: naranjas dulces, definidas como la principal especie con mayor área y diversidad, las mandarinas, los limones y limas ácidas, los pomelos, y en último lugar híbridos, como el tangelo y los tangores (Orduz et al., 2009). Las limas ácidas se cultivan en regiones tropicales y subtropicales de Asia y América, teniendo en las regiones productoras una gran importancia económica; y son un componente fundamental en las cocinas de Asia y Suramérica (Donkersley et al., 2018). Los principales países productores son India, China y México.

La producción de lima ácida Tahití en Estados Unidos se terminó en el año 2006, debido a graves problemas sanitarios. Esta situación ha incentivado la producción en México, Brasil y Costa Rica orientada en gran parte a la exportación a Estados Unidos (Donkersley et al., 2018); como también a Europa. Colombia ha incrementado el área plantada de lima ácida Tahití, así como los volúmenes exportados a ambos mercados.

El área de los cítricos en Colombia es de 96 mil hectáreas cultivadas (MADR, 2017); mientras que para las limas ácidas (Tahití y el pajarito o de Castilla), la Encuesta Nacional Agropecuaria-ENA (DANE, 2017) reporta que existen 23.638 hectáreas en 26 departamentos. De lima ácida Tahití se tienen 10,489 hectáreas (DANE, 2016), estando los departamentos de Tolima, Santander y Valle del Cauca, dentro de los de mayor área y quienes están liderando la exportación de esta fruta cítrica. Se calcula que anualmente se producen alrededor de 100 a 120 mil toneladas de fruta de lima Tahití al año en el país, la producción media de cultivos tecnificados es de $15 \mathrm{t} \mathrm{ha}^{-1}$ pero se han obtenido rendimientos de $40 \mathrm{t}$ ha ${ }^{-1}$ producciones excepcionales de 60-70 $\mathrm{t} \mathrm{ha}^{-1}$ en los cultivos mejor manejados. La lima ácida Tahití puede producir varias floraciones al año y por tanto 2 a 3 cosechas importantes, lo que explica la obtención de esos altos rendimientos.

Para la obtención de óptimos niveles de producción y calidad en la lima ácida Tahití es necesario suplir los requerimientos de riego y una suficiente y oportuna aplicación de nutrientes (Levy y Syvertsen, 2004). Dentro de las principales limitantes para el manejo tecnificado de los cultivos frutales en el país, se debe a la deficiente planeación en lo relacionado con el manejo de los cultivos y en particular de la fertilización. Esta práctica está directamente relacionada con los altos rendimientos de los cultivos y con la calidad de las frutas; sin embargo para determinar fuentes, dosis y épocas no se utilizan los análisis edáficos y foliares específicos para la localidad y variedad, sino que prevalece la práctica cultural tradicional de generalizar las recomendaciones para cítricos en general, lo que finalmente se ve reflejado en bajos rendimientos (Corporación Universitaria Lasallista, 2012) y bajas rentabilidades. El estado nutrimental del cultivo es uno de los factores que determina la cantidad y calidad de los frutos, por ello es necesario conocer qué elementos se encuentran en niveles limitantes en el suelo (Lucena, 1997).

La nutrición de los cultivos busca cubrir la diferencia entre la demanda de la planta para optimizar el rendimiento y la capacidad que tiene el suelo de suministrar los elementos de importancia; sin embargo, una parte de los nutrientes aplicados en la fertilización se pierden por volatilización, lixiviación, fijación y erosión, disminuyendo la eficiencia de esta práctica (Malavolta, 1995). Para tener información precisa del requerimiento nutrimental de los árboles frutales, así como las 
dosis y épocas de aplicación de fertilizantes, es necesario conocer la distribución de la materia seca y la extracción de nutrimentos por los diferentes órganos (Contreras et al., 2007).

Al respecto, se han propuesto relaciones para estimar las dosis de fertilizantes requeridas por un cultivo, considerando la demanda de nutrimentos de los diferentes órganos de la planta, el suministro del suelo y la eficiencia del fertilizante, en las condiciones específicas de manejodelcultivo(Etchevers, 1987; Szucs, 1997).

El análisis de suelos puede usarse conjuntamente con observaciones de campo y nivel de rendimiento para determinar desordenes nutricionales, para controlar los niveles de nutrientes relativamente inmóviles como $\mathrm{Mg}$, $\mathrm{Ca}, \mathrm{Cu}$ y $\mathrm{P}$, y para monitorear el pH (Davies y Albrigo, 1994). El análisis foliar por otra parte, es el método más adecuado para diagnosticar el estado nutricional de la plantación y evaluar la disponibilidad de reservas de la planta (Cohen, 1983), dado que las hojas captan nutrientes durante un tiempo prolongado y el árbol es el sistema de extracción más preciso para indicar la idoneidad de cada nutriente y los desbalances existentes (Davies y Albrigo, 1994).

Con el fin de interpretar los análisis nutrimentales de tejido foliar, con una base científica para mejorar la recomendación de fertilizantes, se han propuesto distintos métodos. En primer lugar, se destaca el Sistema Integrado de Diagnóstico y Recomendación (DRIS), que es un método que utiliza relaciones entre nutrimentos para la interpretación del análisis del tejido en lugar de concentraciones absolutas e individuales (Walworth y Sumner, 1987). El DRIS usa índices para cada nutriente, obtenidos de la media aritmética de las funciones calculadas, considerando las relaciones de todas las parejas de elementos en las que interviene el elemento determinado, de allí que los índices negativos indican deficiencias y los positivos excesos de los elementos analizados (Ventura et al., 2012).
El método convencional o Rango de Suficiencia (RS) sebasa en una recopilación de valores contra los cuales se comparan los resultados obtenidos en laboratorio; este procedimiento asume que se conocen los niveles óptimos, deficientes o excesivos de los nutrimentos. La Desviación del Óptimo Porcentual (DOP) es un método estadístico que compara la concentración del nutriente en la muestra respecto a la norma, la cual se basa en un nivel nutrimental óptimo en la que el cultivo expresa su máximo rendimiento potencial (Ventura et al., 2012). El método DOP cuantifica el valor en que un nutriente se desvía con respecto a la norma individual, por lo cual una situación nutrimental óptima, para cualquier elemento, es definida por el índice DOP igual a cero, dando el orden de limitación tanto por exceso (DOP $>0$ ) como por déficit (DOP $<0$ ), de cada uno de los elementos (Lucena, 1997). El análisis DOP permite conocer directamente el orden relativo de limitación u Orden de Requerimiento Nutricional (ORN) entre los elementos considerados, con base en el cual es posible ajustar las necesidades de fertilización (Montañés et al., 1991).

Por lo anterior, el objetivo de este estudio fue realizar la evaluación nutricional de los cultivos de lima Tahití (Citrus latifolia Tanaka) en las condiciones edafoclímaticas del Tolima, mediante dos métodos de interpretación de análisis foliar (RS y DOP) y análisis de muestras de suelo, para obtener un diagnóstico confiable de la nutrición de esta plantación a escala local.

\section{MATERIALES Y MÉTODOS}

Las muestras para determinación de concentración en tejido foliar y de niveles edáficos de nutrientes fueron tomados en 20 fincas con cultivos de lima Tahití que superaban los tres años en siete municipios del departamento del Tolima (Espinal, Flandes, Coello, Guamo, San Luis, y Armero-Guayabal).

Las muestras para el análisis foliar fueron 
tomadas de brotes no fructíferos de 24 árboles, en ramas a media altura del árbol y se colectaron 4 hojas por planta, una por cada punto cardinal, según lo reportado por Koo et al. (1985) y Malavolta (1995). Mediante el método de interpretación de rango de suficiencia (RS) se realizó la comparación de los resultados analíticos del laboratorio con los de referencia para el cultivo (Jones et al. 1991). La recopilación del RS para más de 300 cultivos realizada por Jones et al. (1991) no indica las variedades ni las condiciones de suelo y clima en que se desarrollaron los cultivos, por lo que sólo es útil como referencia

Esta interpretación implica una comparación directa entre la concentración de un solo elemento y su respectiva norma, por lo cual se dice que es un método estático (Ventura et al., 2012). Los resultados obtenidos del análisis foliar para cada elemento y para cada árbol muestreado fueron promediados y luego comparados directamente con el rango de suficiencia establecido para cada nutriente, de esta manera se determinó para cada uno, si se encontraba dentro de la concentración óptima, en deficiencia o en exceso en la planta. Con base en estos resultados fue posible conocer el orden relativo de limitación entre los elementos considerados u Orden de Requerimiento Nutricional (ORN).

El índice DOP se define como la desviación porcentual de la concentración de un elemento con respecto a la concentración óptima, considerada como valor de referencia (Montañés et al., 1991). Dado que el valor de referencia está representado por intervalos de concentración de los elementos, se estableció como óptimo el valor medio de cada intervalo de suficiencia, según lo referenciado en el estudio realizado por Montañés et al. (1993). El índice DOP para cada elemento y para cada árbol muestreado se calculó aplicado la siguiente relación:

$$
\mathrm{DOP}=\frac{\mathrm{C} \times 100}{\text { Cref }}-100
$$

Donde,

C: corresponde a la concentración foliar del elemento (\% de materia seca) en la muestra analizada.

Cref: es la concentración óptima del mismo elemento (\% de materia seca).

Con base en el signo de los índices obtenidos se pudo determinar si el elemento se encontraba en exceso o en deficiencia. Al igual que mediante el método RS, también se obtuvo el ORN.

Para la interpretación de análisis de suelo se calculó el promedio de cada parámetro fisicoquímico (numérico) considerado, obteniendo de esta manera el promedio de $\mathrm{pH}$, contenido de materia orgánica, fósforo, azufre, calcio, magnesio, potasio, capacidad de intercambio catiónico efectiva, boro, cobre, hierro, manganeso y zinc.

Adicionalmente, se calcularon las relaciones catiónicas promedio de las muestras de suelo colectadas en 20 fincas y fueron comparadas con valores óptimos reportados por Gómez (2005) y Castro y Gómez (2010).0).

Con base en los análisis realizados y la interpretación de estos por diferentes métodos, se establecieron relaciones de causalidad para emitir un diagnostico nutricional acertado para el cultivo de lima ácida en esta localidad.

\section{RESULTADOS Y DISCUSIÓN}

En la tabla 1 se presentan las medidas descriptivas dispersión de los resultados de las muestras foliares de los tejidos evaluados. Para el caso de los macronutrientes: P, Mg y $\mathrm{S}$ la dispersión en la concentración foliar es relativamente baja, en comparación con $\mathrm{N}, \mathrm{K}$ y $\mathrm{Ca}$, los cuales presentan una mayor variación. Pese a su alta variabilidad, el Ca es el nutriente de mayor concentración aún 
por encima de $\mathrm{N}$, siendo este el nutriente necesario en mayor proporción en las partes vegetativas de las plantas (Mattos et al., 2010).

ParaelcasodemicronutrienteselCueselelemento de mayor variabilidad dentro de la población, mostrando un valor máximo de casi $250 \mathrm{mg} \mathrm{kg}^{-1}$.

Se observa un comportamiento similar para los contenidosdeZnyB, concantidadesquealcanzan casi los 140 y $810 \mathrm{mg} \mathrm{kg}^{-1}$, respectivamente.

Valores extremos en las concentraciones de nutrientes en tejido foliar afectan señalan una mayor diferencia en la nutrición de los cultivos debidas probablemente a la variabilidad natural entre la fertilidad de los suelos utilizados para el cultivo, los usos previos o en las diferencias en el manejo de los cultivos debido al nivel tecnológico, o a la utilización de tecnología para las recomendaciones de fertilización. entre la fertilidad de los suelos utilizados para el cultivo, los usos previos o en las diferencias en el manejo de los cultivos debido al nivel tecnológico, o a la utilización de tecnología para las recomendaciones de fertilización.

Tabla 1. Medidas descriptivas de contenido de nutrientes en tejido foliar tomadas del cultivo de lima Tahití en siete municipios del departamento del Tolima (Espinal, Flandes, Coello, Guamo, San Luis, y Armero-Guayabal).

\begin{tabular}{lrrrrrrr}
\hline Elemento & Máximo & Mínimo & Media & Mediana & Varianza & $\begin{array}{c}\text { Desviación } \\
\text { estándar }\end{array}$ & $\begin{array}{c}\text { Coeficiente de } \\
\text { variación de la } \\
\text { media }\end{array}$ \\
\hline $\mathrm{N}(\%)$ & 2,79 & 1,92 & 2,46 & 2,52 & 10,07 & 10,07 & 10,37 \\
$\mathrm{P}(\%)$ & 0,30 & 0,16 & 0,26 & 0,21 & 0,001 & 0,001 & 16,20 \\
$\mathrm{~K}(\%)$ & 2,11 & 0,73 & 1,36 & 1,42 & 0,149 & 0,149 & 28,32 \\
$\mathrm{Ca}(\%)$ & 5,38 & 2,63 & 3,78 & 3,52 & 0,613 & 0,613 & 20,70 \\
$\mathrm{Mg}(\%)$ & 0,53 & 0,15 & 0,29 & 0,26 & 0,007 & 0,007 & 30,38 \\
$\mathrm{~S}(\%)$ & 0,36 & 0,19 & 0,23 & 0,22 & 0,001 & 0,001 & 16,32 \\
$\mathrm{Fe}\left(\mathrm{mg} \mathrm{kg}{ }^{-1}\right)$ & 890,00 & 196,00 & 340,86 & 331,50 & 19039,94 & 19039,94 & 40,48 \\
$\mathrm{Cu}\left(\mathrm{mg} \mathrm{kg}^{-1}\right)$ & 255,00 & 3 & 58,75 & 21,50 & 6366,02 & 6366,02 & 135,81 \\
Mn (mg kg-1) & 93,00 & 8 & 48,36 & 43,50 & 604,16 & 604,16 & 50,81 \\
$\mathrm{Zn}\left(\mathrm{mg} \mathrm{kg}^{-1}\right)$ & 137,00 & 5 & 37,33 & 24,00 & 1462,58 & 1462,58 & 102,44 \\
$\mathrm{~B}\left(\mathrm{mg} \mathrm{kg}^{-1}\right)$ & 808,99 & 108,42 & 243,88 & 166,31 & 37597,14 & 37597,14 & 79,1 \\
\hline
\end{tabular}

Para la interpretación de los datos analíticos y el diagnóstico exacto de la situación nutricional del cultivo, se disponen de valores de referencia u óptimos nutricionales. La tabla 2 presenta las concentraciones promedio de cada uno de los elementos analizados en las muestras de tejido foliar y los rangos de suficiencia para cada nutriente (Malavolta, 1995).
Para macronutrientes, las concentraciones se encuentran muy cerca o dentro del rango óptimo de cada elemento, con excepción del $\mathrm{P}$ que exhibe valores superiores y el Mg inferiores al intervalo óptimo. Mostrando con más detalle el estado nutricional del cultivo la figura $2 \mathrm{~A}$ ilustra la proporción de plantas muestreadas con deficiencias, excesos y con los contenidos

Tabla 1. Concentración promedio y rangos de suficiencia para elementos nutricionales en muestras de tejido foliar en el cultivo de lima ácida Tahití en siete municipios del departamento del Tolima (Espinal, Flandes, Coello, Guamo, San Luis, y Armero-Guayabal), 2014.

\begin{tabular}{|c|c|c|c|c|c|c|c|c|c|c|c|}
\hline Elemento & $\mathbf{N}$ & $\mathbf{P}$ & $\mathbf{K}$ & $\mathrm{Ca}$ & $\mathrm{Mg}$ & $S$ & $\mathrm{Fe}$ & $\mathrm{Cu}$ & $\begin{array}{c}\text { Mn } \\
\mathrm{mg} \mathrm{kg}^{-1}\end{array}$ & $\mathrm{Zn}$ & $\mathrm{Zn}$ \\
\hline $\begin{array}{l}\text { Concentración } \\
\text { foliar promedio }\end{array}$ & 2,46 & 0,21 & 1,36 & 3,77 & 0,28 & 0,23 & 340,88 & 58,75 & 48,38 & 37,33 & 243,88 \\
\hline Rangos óptimos & $2,5-2,7$ & $0,12-0,17$ & $1,2-1,7$ & $3,0-4,5$ & $0,3-0,5$ & $0,2-0,4$ & $50-120$ & $5-12$ & $25-49$ & $25-49$ & $35-100$ \\
\hline
\end{tabular}




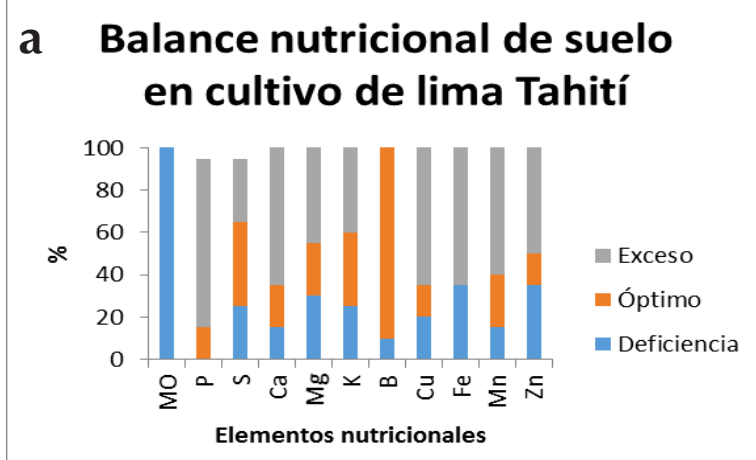

\section{b Balance Nutricional en Tejido Foliar de Cultivo de Lima Tahití}

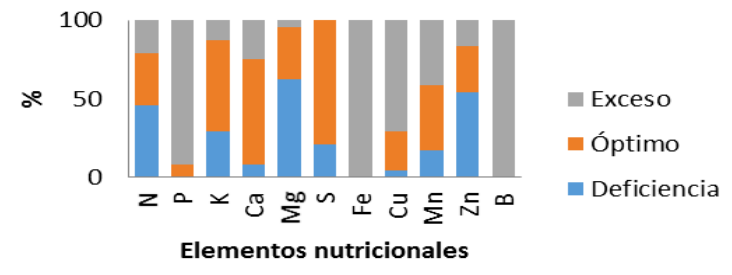

Figura 2. Proporción de la población de árboles de lima Tahití muestreados en estado de deficiencia, óptimo y de exceso de cada elemento nutricional de acuerdo con los rangos de suficiencia para elementos nutricionales en muestras de tejido foliar (izquierda) y de suelo (derecha) en siete municipios del departamento del Tolima (Espinal, Flandes, Coello, Guamo, San Luis, y Armero-Guayabal), 2014. .

adecuados de cada uno de los elementos. Con base en la interpretación del análisis foliar usando el método de rango de suficiencia se logró establecer el ORN así: $\mathrm{Mg}>\mathrm{Zn}>\mathrm{N}>\mathrm{K}>\mathrm{S}>\mathrm{Mn}>\mathrm{Ca}>\mathrm{Cu}$, es decir, que las deficiencias nutricionales se presentaron en altas proporciones dentro de la población muestreada para el Mg y en bajas proporciones para el $\mathrm{Cu}$.

En la tabla 1 se muestra al $\mathrm{Zn}$ dentro del rango óptimo, mientras que en la figura $2 \mathrm{~A}$ la proporción de plantas con deficiencias de este elemento es alta, este comportamiento obedece a la influencia sobre el contenido promedio de valores extremos relacionados con exceso de $\mathrm{Zn}$ en algunos árboles muestreados.

El ORN sugiere, modificar el plan de fertilización de modo que la proporción de plantas dentro del rango óptimo sea mayor, tomando en cuenta el ajuste necesario para los elementos que se encuentran en concentraciones excesivas dentro de la planta, que son en orden descendente $\mathrm{Fe}, \mathrm{B}, \mathrm{P}, \mathrm{Cu}$, Mn y en menor proporción $\mathrm{Ca}, \mathrm{N}, \mathrm{Zn}, \mathrm{K}$ y Mg.

Deficiencias de Mg, que son las más frecuentes dentro de la población de árboles muestreada, resultan en desbalances nutricionales que en cítricos se evidencian en moteados cloróticos que se extienden desde la base hasta el ápice de la hoja, en ambos lados de la nervadura central en hojas maduras, que luego tienden a convertirse en manchas necróticas (Saha, 2002, Srivastava et al., 2015). Lo anterior como consecuencia de la afectación de deficiencia de magnesio sobre procesos fisiológicos como la fotosíntesis (Tang et al., 2012), glicólisis y el ciclo de ácidos tricarboxílicos (Yang et al., 2013).

La condición de deficiencia de Mg en 62,5\% de los árboles muestreados podría estar asociada al $\mathrm{pH}$ del suelo, aunque en promedio se tuvo un $\mathrm{pH}$ de 5,8 (moderadamente ácido), en condiciones alcalinas, la formación de $\mathrm{MgCO}_{3}$ y el exceso de $\mathrm{Ca}, \mathrm{K}$ y $\mathrm{Na}$, pueden reducir la disponibilidad del magnesio para los cultivos (Broadley y White, 2010).

De otra parte, se presenta exceso de elementos como cobre, llegando a niveles tóxicos para las plantas. Para la mayoría de especies cultivadas, el nivel crítico de toxicidad de $\mathrm{Cu}$ en hojas se encuentra por encima de 20 a 30 mg kg-1 , lo que generalmente causa inhibición del crecimiento radical (Robson y Reuter, 1981).

La situación nutricional evidenciada a través de la desviación del óptimo porcentual (DOP) se ilustra en la figura 3. Se presentan los índices en orden ascendente, los valores negativos representan deficiencia y valores positivos representan excesos. 


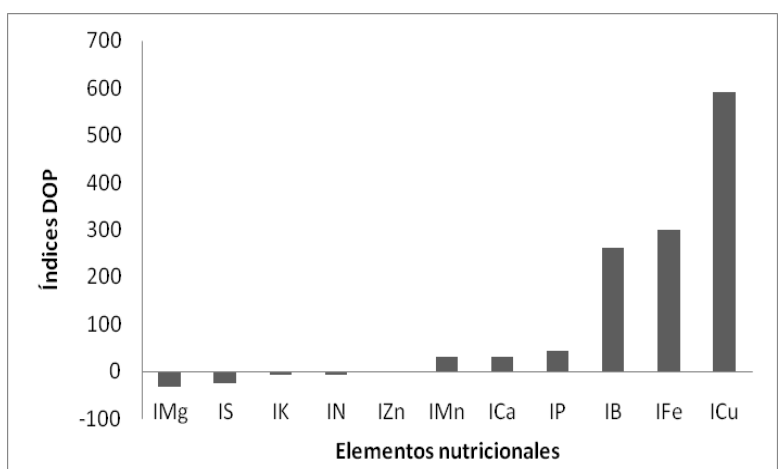

Figura 3. Índices de desbalance para cada nutriente en muestras foliares de cultivo de lima ácida Tahití, con base en cálculos DOP..

Se dan excesos marcados de $\mathrm{Cu}$ y deficiencias de Mg. Con base en estos valores, el ORN se establece así: $\mathrm{Mg}>\mathrm{S}>\mathrm{K}>\mathrm{N}$. El índice DOP del $Z n$ es de 0.9 , lo cual sugiere que se encuentra en concentraciones óptimas dentro del árbol. $\mathrm{Mn}, \mathrm{Ca}$ y $\mathrm{P}$ presentan ligeros excesos y $\mathrm{B}, \mathrm{Fe}$ y $\mathrm{Cu}$ muestran elevados contenidos dentro de la planta, sugiriendo niveles de toxicidad.

El ORN obtenido mediante ambos métodos es similar, aunque DOP no refleja deficiencias marcadas de Zn. Lo anterior sugiere que DOP es menos susceptible a la presencia de valores extremos para las concentraciones foliares de elementos nutricionales, y por tanto una herramienta más adecuada sobre la cual ajustar niveles de fertilización para los cultivos.

Las medidas descriptivas de los niveles de macro y micronutrientes en suelo se presentan en la tabla 3. Se observa que la variación en los contenidos de $\mathrm{P}$ es alta. De igual manera sucede para Fe, lo anterior sugiere desbalances para dichos elementos dentro de la población muestreada.

Tabla 3. Medidas descriptivas de contenido de nutrientes en suelo del cultivo de lima Tahití en siete municipios del departamento del Tolima (Espinal, Flandes, Coello, Guamo, San Luis, y Armero-Guayabal).

\begin{tabular}{crrrrrrr}
\hline Elemento & Máximo & Mínimo & Media & Mediana & Varianza & $\begin{array}{c}\text { Desviación } \\
\text { estándar }\end{array}$ & $\begin{array}{c}\text { Coeficiente de } \\
\text { variación de la } \\
\text { media }\end{array}$ \\
\hline MO & 2,75 & 0,57 & 1,45 & 1,25 & 0,43 & 0,66 & 45,33 \\
$\mathrm{P}$ & 316,00 & 14,60 & 94,84 & 85,70 & 6529,99 & 80,81 & 85,21 \\
$\mathrm{~S}$ & 98,40 & 6,00 & 27,04 & 12,20 & 849,99 & 29,16 & 107,81 \\
$\mathrm{Ca}$ & 23,27 & 1,35 & 5,99 & 4,46 & 32,99 & 5,74 & 95,96 \\
$\mathrm{Mg}$ & 2,34 & 0,41 & 1,16 & 1,08 & 0,31 & 0,55 & 47,48 \\
$\mathrm{~K}$ & 0,90 & 0,18 & 0,45 & 0,39 & 0,05 & 0,23 & 50,43 \\
$\mathrm{~B}$ & 0,75 & 0,22 & 0,41 & 0,41 & 0,02 & 0,14 & 33,32 \\
$\mathrm{Cu}$ & 34,00 & 0,60 & 5,78 & 2,80 & 66,98 & 8,18 & 141,71 \\
$\mathrm{Fe}$ & 592,00 & 7,50 & 80.37 & 60,55 & 15441,55 & 124,26 & 154,63 \\
$\mathrm{Mn}$ & 55,00 & 3,70 & 18.71 & 13,65 & 233,04 & 15,27 & 81,61 \\
$\mathrm{Zn}$ & 30,00 & 0,80 & 7,89 & 5,40 & 65,21 & 8,08 & 102,41 \\
\hline
\end{tabular}

La tabla 4 presenta los valores promedio para los niveles edáficos de los nutrientes y los rangos óptimos reportados para cada elemento. Se evidencia como mayor limitante el contenido de MO orgánica en el suelo, el cual se encuentra por debajo del rango adecuado. Los elementos restantes se encuentran en su mayoría ligeramente superiores al intervalo óptimo. La conductividad eléctrica (CE) reportada en el análisis de las muestras de suelo, no representa limitaciones para el desarrollo del cultivo.
La Figura 2B ilustra el balance nutricional del suelo en el cultivo de lima Tahití, con base en los rangos de suficiencia de la Tabla 2.

Se destaca que el $100 \%$ de las muestras de suelo colectadas presentan deficiencias en los contenidos de $\mathrm{MO}$ y por tanto de $\mathrm{N}$. en términos generales, ningún elemento se encuentra en deficiencia significativa dentro de la población muestreada. De otro lado, se evidencia exceso marcado en el nivel de $\mathrm{P}, \mathrm{Ca}$, 
Tabla 4. Valores promedio de parámetros fisicoquímicos obtenidos a partir de análisis de suelo en el cultivo de lima ácida Tahití en siete municipios del departamento del Tolima (Espinal, Flandes, Coello, Guamo, San Luis, y Armero-Guayabal), 2014.

\begin{tabular}{|c|c|c|c|c|c|c|c|c|c|c|c|c|c|c|}
\hline Parámetro & pH & $\begin{array}{c}\text { Ce } \\
\text { dS } \text { m }^{-1}\end{array}$ & $\begin{array}{c}\text { MO } \\
\%\end{array}$ & $\begin{array}{l}\mathrm{P} \\
\mathrm{mg}\end{array}$ & $\begin{array}{r}S \\
K^{-1}\end{array}$ & $\mathrm{Ca}$ & $\begin{array}{c}\mathrm{Mg} \\
\mathrm{cmol} \mathrm{Kg}\end{array}$ & K & $\mathrm{Na}$ & B & $\begin{array}{l}\mathrm{Cu} \\
\mathrm{mg}\end{array}$ & $\begin{array}{r}\mathrm{Fe} \\
\mathrm{kg}^{-1}\end{array}$ & $M n$ & $\mathbf{Z n}$ \\
\hline $\begin{array}{l}\text { Concentración } \\
\text { foliar promedio }\end{array}$ & 5,87 & 0,41 & 1,45 & 85,7 & 12,2 & 4,46 & 1,08 & 0,39 & 0,24 & 0,41 & 2,8 & 60,55 & 13,65 & 5,4 \\
\hline Rangos óptimos & $4.5-6.0$ & & $4-8$ & $10-20$ & $10-15$ & $2-3$ & $0,8-1,2$ & $0,3-0,4$ & & $0,3-1$ & $1-1,5$ & $40-50$ & $5-10$ & $3-5$ \\
\hline
\end{tabular}

$\mathrm{Cu}, \mathrm{Fe}, \mathrm{Mn}$ y $\mathrm{Zn}$. En el $90 \%$ de las muestras colectadas, los niveles de boro se encuentran dentro del rango adecuado para este elemento.

El pH en la solución presenta un valor promedio de 5,87, sin embargo, los valores obtenidos en laboratorio oscilan entre 4,7 (muy fuertemente ácidos) y 8,3 (moderadamente alcalino), esto puede ejercer un efecto directo sobre los cultivos, ya que, a una determinada acidez o alcalinidad, los elementos nutritivos son más o menos asimilables. Los excesos de hierro y manganeso pueden presentarse en suelos con $\mathrm{pH}$ bajo, tomando en cuenta que los metales generalmente están fuertemente unidos a las partículas de suelo o existen naturalmente como minerales sólidos a un pH alto (Castro y Gómez, 2010).

Observado los resultados de los análisis de tejido foliar y de suelo en el cultivo de lima Tahití, es posible observar que para algunos casos la disponibilidad de nutrientes en el suelo repercute de manera directa sobre los contenidos de los mismos en el tejido foliar de la planta, particularmente para el $\mathrm{P}, \mathrm{Fe}, \mathrm{Cu}$ y $\mathrm{Mn}$, se presentaron excesos tanto en niveles edáficos como en concentraciones foliares. No se evidenció muy claramente esta relación directa para los otros nutrientes, dado que los contenidos foliares de los nutrientes no dependen únicamente de la disponibilidad del elemento en el suelo, pues se ven influenciados por otros factores como la tasa de crecimiento de la planta, la edad de la hoja, las combinaciones copa-patrón y las interacciones entre los mismos nutrientes (Mattos et al., 2010).

\section{CONCLUSIONES}

Las dos técnicas de interpretación de análisis de tejido foliar de árboles de lima Tahití, mostraron resultados similares en cuanto a ORN; sin embargo, se considera a la técnica DOP como la más precisa para emitir diagnósticos de nutrición de cultivos al no verse influenciada por valores extremos de concentración foliar de elementos dentro de la población de plantas muestreada.

Se presentó una relación directa entre el nivel edáfico y la respectiva concentración foliar para algunos nutrientes, lo cual sugiere que el uso de análisis de muestras foliares y de suelo, se constituyen como herramientas importantes que en conjunto pueden brindar información más acertada sobre la situación nutricional del cultivo de lima Tahití. Con base en estos indicadores, es posible reestructurar planes de manejo de fertilización en este sistema de producción.

\section{AGRADECIMIENTOS}

Al Ministerio de Agricultura y Desarrollo Rural por la financiación de la investigación para realizar el diagnóstico del "Wood Pocket" en la citricultura del Tolima ejecutado por Corpoica en el año 2011.

\section{REFERENCIAS}

Broadley, M., Brown, P., Calmal, I., Rengel, Z. y Zhao, F. 2012. Function of nutrients: micronutrients. En:Marshner, P. Marschner's Mineral Nutrition of Higher Plants. Terceraedición. University of Adelaide. Australia. 191- 248 p. 
Broadley, M. y White, P. 2010. Eats roots and leaves. Can edible horticultural crops address dietary calcium, magnesium and potassium deficiencies? Proc Nutr Soc 69, 601-612. https://doi. org/10.1017/S0029665110001588

Castro, H y Gómez, M. 2010. Fertilidad de suelos y fertilizantes. En: Ciencia del suelo Principios básicos. Sociedad Colombiana de la Ciencia del Suelo. 217- 298pp.

Cohen, A. 1983. Fertilización de los cítricos. Inter. Potasa Institute. Bull. (4): 12-18

Contreras, E., Almaguer, G., Espinoza, J., Maldonado, R. y Álvarez, E. 2007. Distribución de materia seca y nutrimentos en árboles de limón persa (Citrus latifolia Tan.) en Veracruz, México. Revista Chapingo. Serie Horticultura. 13(1), 77- 85.

Corporación Universitaria Lasallista (Ed.). 2012. Cítricos: Cultivo, Poscosecha e Industrialización. Serie Lasallista Investigación y Ciencia. Artes y Letras S.A.S. Itagüí, Colombia. 367 p.

Davies, F. y Albrigo, L. 1994. Citrus. CAB International, Wallingford, U.K. 254 p.

DANE. Departamento Administrativo Nacional de Estadística 2016. Encuesta Nacional Agropecuaria-ENA. Disponible en: https://www.dane.gov.co/index. php/estadisticas-por-ena/agropecuario/ encuesta-nacional-agropecuariaena. Consulta 30 de marzo 2018

Donkersley, P., Silva, F. W., Carvalho, C. M., AlSadi, A. M., \& Elliot, S. L. 2018. Biological, environmental and socioeconomic threats to citrus lime production. Journal of Plant Diseases and Protection, 1-18.

Etchevers B. 1987. Diagnóstico visual. Centro de edafología. Colegio de Postgraduados. Chapingo, Estado de México.
Gómez, M. 2005. Análisis de suelos como herramienta de diagnóstico en la evaluación química de la fertilidad en el cultivo de papa. En: Fisiología y nutrición vegetal en el cultivo de papa. Presente y futuro de la investigación en la cadena agroalimentaria de la papa en Colombia. CEVIPAPA. Bogotá, Colombia. 99p.

Jackson, L. K. 1999. Citrus cultivation. Citrus Health Management. Timmer, LW and LW Duncan. (eds.) pp, 21-46.

Jones, B., Wolf, B. and H. A. Mills. 1991. Plant analysis handbook: a practical sampling, preparation, analysis, and interpretation guide. MicroMacro Publishing. Athens, GA, USA.

Levy Y, Syvertsen J. 2004. Irrigation water quality and salinity effects in citrus trees. Hortic Rev 30:37-82

Lucena, J. 1997. Methods of diagnosis of mineral nutrition of plant a critical review. Acta Hortic. 448, 179-192p. https://doi. org/10.17660/ActaHortic.1997.448.28

Malavolta, E. 1995. Nutrición y fertilización de los cítricos. Fertilización de cultivos de clima medio. $2^{\text {a }}$ edición. Monómeros Colombo-Venezolanos, Bogotá, 133-169.

Mattos, D., Milaneze, T., Alves, F. y Quaggio, J. 2010. Soil nutrient availability and its impact on fruit quality of Tahiti acid lime. Rev. Bras. Frutic. Jaboticabal. 32(1), 335- 342. https://doi.org/10.1590/ S $0100-29452010005000032$

Montañés, L., Heras, L. y Sanz, M. 1991. Desviación del Óptimo Porcentual (DOP): Nuevo índice para la interpretación del análisis vegetal. An. Aula Dei. 20(3-4), 93- 107. https://doi. org/10.1080/01904169309364613

Montañés, L., Heras, L., Abadía, J. y Sanz, M. 1993. Plant analysis interpretation based on a new index: Deviation from optimum percentage (DOP). J. Plant Nutr. 16, 1289- 1308

\section{Ministerio de Agricultura y Desarrollo Rural, 2017. Evaluaciones Agropecuarias Municipales-EVA.}


Orduz Rodríguez, J., León, G. y Arango, L. 2009. Lima Ácida Tahiti: Opción agrícola para los Llanos Orientales de Colombia. Corpoica, Issue 53, p. 5.

Orduz-Rodriguez, J. 1998. Características de la citricultura en el departamento del Meta y avances en el proceso de desarrollo tecnológico. En: Actualización tecnológica en ajonjolí, caucho, hortalizas y frutales para la Orinoquía colombiana. Corpoica. Villavicencio,

Quiroga, J., Hernández, F., Silva, M. y OrduzRodriguez, J. 2010. Comportamiento de la producción de lima Tahití (Citrus latifolia Tanaka), injertada sobre el patrón de mandarina Cleopatra (Citrus reticulata Blanco) y la influencia del virus de la tristeza (CTV) en condiciones del piedemonte del Meta, 1997-2008. Orinoquia. 14(1), 1-11.

Robson, A. y Reuter, D. 1981. Diagnosis of copper deficiency and toxicity. En: J. F. Loneragan, A. D. Robson and R. D. Graham (Eds.). Copper in Soils and Plants. Academic Press, London and Orlando. 287-312 pp.

Saha, L. 2002. Hand book of plant diseases. KalyaniPublishers, Ludhiana,pp290-292.

Srivastava, J., Sharma, P., Dutta, U., Srivastava, A. y Kumar, R. 2015. Nutritional disorders of citrus and their management. En: Awashti, L. (Ed.). Recent advances in ths diagnosis and management of plant diseases. Springer. India. 285- 294 pp. https://doi. org/10.1007/978-81-322-2571-3_21
Szucs, H. 1997. Possibilities to meet nutritional requirements of fruit trees and environmental protection. Acta Horticulture. 448, 433-436. https://doi. org/10.17660/ActaHortic.1997.448.80

Tang, N., Li, Y. y Chen, Li. 2012. Magnesium deficiency-induced impairment of photosynthesis in leaves of fruiting Citrus reticulata trees accompanied by upregulation of antioxidant metabolism to avoid photo-oxidative damage. J. Plant. Nutr. Sci. 175, 784-793. https:// doi.org/10.1002/jpln.201100329

Ventura, F., Salgado, S., Castelán, M., Palma, D., Rivera, M. y Sánchez, P. 2012. Métodos de interpretación del análisis nutrimental en naranja valencia (Citrus sinensis L. Osbeck). Terra Latinoamericana. 30(2), 139- 145.

Walworth, J. y Sumner, M. 1987. The diagnosis and recommendation integrated system (DRIS). Advances in Soil Science. 6, 149-188. https://doi. org/10.1007/978-1-4612-4682-4_4

Yang, L., Yang, G., You, X., Zhou, C., Lu, Y.y Chen, L. 2013. Magnesium deficiency-induced changes in organic acid metabolism of Citrus sinensis roots and leaves. Biology Plantarum 57(3), 481- 486. https:// doi.org/10.1007/s10535-013-0313-5 